\title{
Assessment of quality of life in people with severe and enduring anorexia nervosa: a comparison of generic and specific instruments
}

\author{
Deborah Mitchison ${ }^{1 *}$, Phillipa Hay ${ }^{2,3}$, Scott Engel ${ }^{4}$, Ross Crosby ${ }^{4}$, Daniel Le Grange ${ }^{5}$, Hubert Lacey ${ }^{6}$, \\ Jonathan Mond ${ }^{7,8}$, Shameran Slewa-Younan ${ }^{9}$, Stephen Touyz ${ }^{10}$ \\ From 2013 ANZAED Conference: Inspiring Change: Person and Context \\ Melbourne, Australia. 23-24 August 2013
}

\section{Aims}

To compare the psychometric properties of generic and disease-specific measures of health-related quality of life (HRQoL).

\section{Methods}

63 participants with anorexia nervosa (AN) completed measures of generic HRQoL (Medical Outcomes Study Short-Form; SF-12), disease-specific HRQoL (Eating Disorders Quality of Life Questionnaire; EDQOL), functional impairment (days out of role; Work and Social Adjustment Scale), and eating disorder severity (Eating Disorder Examination Questionnaire) at baseline, posttreatment, and 6-12 months follow-up. The SF-12 and EDQOL were compared on internal consistency, convergence validity, and criterion-related validity.

\section{Results}

The EDQOL had stronger internal consistency $\alpha=0.92$ vs. 0.80), convergence with eating disorder severity $(\mathrm{r}=$ 0.06 to 0.53 vs. 0.11 to -0.39 ), and criterion-related (functional impairment) validity ( $\beta=0.37-0.46$ ) compared to the SF-12. The SF-12 converged more strongly with functional impairment $(\mathrm{r}=-0.31$ to -0.63 vs. 0.06 to 0.70$)$. The SF-12 ( $\beta=0.41-0.42)$ and EDQOL $(\beta=0.33-0.38)$ had similar criterion-related (eating disorder severity) validity.

\section{Conclusions}

The EDQOL and SF-12 should be used where possible in tandem as measures of HRQoL, whereas the SF-12 may be preferable in studies where comparisons are to be made with other populations.

This abstract was presented in the Anorexia Nervosa Characteristics and Treatment stream of the 2013 ANZAED Conference.

\section{Authors' details}

${ }^{1}$ School of Medicine, University of Western Sydney, Australia. ${ }^{2}$ Centre for Health Research, School of Medicine, University of Western Sydney, Australia. ${ }^{3}$ School of Medicine, James Cook University, Australia. ${ }^{4}$ Neuropsychiatric Research Institute, Fargo, School of Medicine and Health Sciences, University of North Dakota, USA. ${ }^{5}$ Department of Psychiatry and Behavioral Neuroscience, University of Chicago, USA. ${ }^{6}$ Division of Population Health Sciences and Education, St George's, University of London, UK. ${ }^{7}$ School of Medicine and Public Health, University of Newcastle, Australia. ${ }^{8}$ School of Sociology, Australian National University, Australia. ${ }^{9}$ School of Medicine, University of Western Sydney, Australia. ${ }^{10}$ School of Psychology, University of Sydney, Australia.

Published: 14 November 2013

doi:10.1186/2050-2974-1-S1-042

Cite this article as: Mitchison et al: Assessment of quality of life in people with severe and enduring anorexia nervosa: a comparison of generic and specific instruments. Journal of Eating Disorders 2013 1(Suppl 1):O42.

* Correspondence: debbie.mitchison@gmail.com

${ }^{1}$ School of Medicine, University of Western Sydney, Australia

Full list of author information is available at the end of the article 